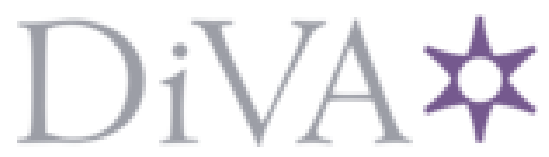

http://www.diva-portal.org

This is the published version of a paper published in SIAM Journal on Scientific Computing.

Citation for the original published paper (version of record):

Tillenius, M. (2015)

SuperGlue: A shared memory framework using data versioning for dependency-aware task-based parallelization.

SIAM Journal on Scientific Computing, 37: C617-C642

http://dx.doi.org/10.1137/140989716

Access to the published version may require subscription.

N.B. When citing this work, cite the original published paper.

Permanent link to this version:

http://urn.kb.se/resolve?urn=urn:nbn:se:uu:diva-266813 


\title{
SUPERGLUE: A SHARED MEMORY FRAMEWORK USING DATA VERSIONING FOR DEPENDENCY-AWARE TASK-BASED PARALLELIZATION*
}

\author{
MARTIN TILLENIUS ${ }^{\dagger}$
}

\begin{abstract}
In computational science, it is necessary to make efficient use of multicore architectures for dealing with complex real-life application problems. However, with increased hardware complexity, the cost in man hours of writing and rewriting software to adapt to evolving computer systems is becoming prohibitive. Task-based parallel programming models aim to allow the application programmers to focus on the algorithms and applications, while the performance is handled by a runtime system that schedules the tasks onto nodes, cores, and accelerators. In this paper we describe a task parallel programming model where dependencies are represented through data versioning. Our model allows expressing the program control flow without artificial dependencies, has low complexity for resolving dependencies, and enables scheduling decisions to be made locally. We implement this as a freely available $\mathrm{C}++$ header-only template library, and show experimental results indicating that our implementation both scales and performs well in comparison to similar runtime systems.
\end{abstract}

Key words. task parallel, data version, dependency, shared memory

AMS subject classifications. $65 \mathrm{Y} 05,65 \mathrm{Y} 10$

DOI. $10.1137 / 140989716$

1. Background and related work. Modern processors for laptop, desktop, and server computers have several computational cores. In order to write efficient software for such processors, software needs to be parallel. Since writing parallel software is known to be difficult and error-prone, it is desirable that the parallelizationspecific parts are separated from the rest of the software.

In this paper, we present a runtime system that handles the details of the parallelization for the user. The runtime system manages dependencies between computations and the mapping of computations to hardware resources for the programmer. Since we specifically target scientific computing applications where performance is key, the provided abstractions are designed carefully not to sacrifice performance. To be practically useful and easy to incorporate in existing solutions, the runtime system is provided as a header-only $\mathrm{C}++$ library and is able to both run on top of OpenMP or to use POSIX threads (Pthreads) for thread management.

By moving the dependency management and scheduling into a library that exposes a convenient and expressive interface for specifying dependencies, the development of parallel software becomes easier, faster, and less error-prone and is likely to result in more efficient software.

1.1. Dependencies and synchronization. The most common way to write shared-memory parallel software is to parallelize for-loops using OpenMP [7]. While

*Submitted to the journal's Software and High-Performance Computing section October 2, 2014; accepted for publication (in revised form) September 11, 2015; published electronically November 10, 2015. This work was carried out within the Linnaeus centre of excellence UPMARC, Uppsala Programming for Multicore Architectures Research Center. It was supported by the Swedish Research Council.

http://www.siam.org/journals/sisc/37-6/98971.html

$\dagger$ Department of Information Technology, Uppsala University, SE-751 05 Uppsala, Sweden (martin. tillenius@it.uu.se).

C617 


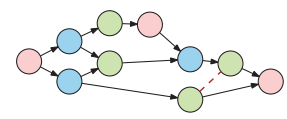

(a) True dependencies

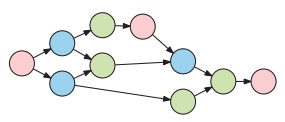

(b) DAG

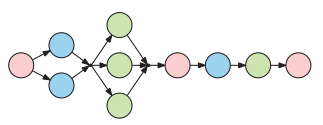

(c) Fork-join

FIG. 1. Examples of how the dependencies in an application can be described. Circles denote tasks and edges denote dependencies. The dashed line in (a) means that the tasks it connects must not execute concurrently.

this works well for many applications, it enforces a fork-join structure where the software is divided up into parallel sections that end with a barrier where all threads are synchronized again. These barriers scale poorly as the number of cores increases and can reduce the performance substantially. To achieve higher performance, synchronization between threads needs to be more fine-grained and reduced to a minimum. To fulfill this, we select a task-based programming model where tasks may have dependencies between them.

A solution to the shortcomings of the fork-join model is to build a directed acyclic graph (DAG) of the tasks, with edges between tasks that have a dependency. This allows more fine-grained dependencies than is possible to express in a fork-join model and reduces unnecessary synchronization points. But not all kinds of dependencies can be represented by a DAG. An edge means that one task must complete before the next is started. In the case of reduction operations, such as accumulating partial results in a shared variable, the tasks may run in any order but must not run concurrently. To describe this kind of dependency in a DAG, either the order of the reduction tasks must be fixed, which limits the scheduler, the graph must be rewritten at runtime, which is expensive, or these dependencies must be left out and handled by some external construct. That is, a static DAG alone cannot describe the true dependencies.

Figure 1 shows the true dependencies of an application and examples of how the dependencies can be expressed in different models. Two of the tasks in this application can run in any order but not at the same time, which is indicated in subfigure (a) by a dashed line connecting them. In the DAG description in subfigure (b), an artificial must-execute-before dependency has been introduced between the tasks instead, and the order in which they must execute has been fixed. In the fork-join model in subfigure (c), two artificial barriers have been introduced.

In applications where parallelism is scarce, introducing artificial dependencies and unnecessary synchronization may be devastating for performance. A goal of this work is to never overestimate dependencies.

1.2. Scheduling. In our system, we consider all processor cores to be identical. We want to maximize throughput and do not consider fairness or task deadlines. Since some applications need to be able to create tasks dynamically at runtime, dependency management and task scheduling must happen at runtime. This means that there is very limited information to use for scheduling; the set of tasks to schedule is not known in advance, and the lengths of the tasks are not known either. Also, tasks are not preemptive. Once the dependencies are met, the only objective we schedule for is locality. That is, tasks that access the same data should preferably execute on the same core, in order to reuse data already present in the caches of that core.

Among the most important properties of the scheduler is that it is fast. In some applications, it is hard to extract parallelism without making the tasks very small. If the time spent in the runtime system between tasks is not negligible compared with 
the time spent in the tasks, this will limit scalability and can prevent applications with fine-grained tasks from scaling at all. This means that the overhead and the time spent on scheduling must be minimized.

The scheduling needs to scale to large numbers of cores. This requires communication between cores to be avoided, and that no central information is needed for scheduling. Scheduling decisions should as far as possible be made locally on each thread.

1.3. Contributions. In this paper, we describe an alternative and flexible way to represent dependencies, using data versions instead of dependency graphs. Tasks in this model depend only on data but not on other tasks. Tasks are queued at the data they need, which avoids central synchronization and leads to locality driven scheduling.

The runtime system and the way dependencies are represented were developed in previous work [24, 23]. In this paper, we further develop the programming model, present new experiments with improved performance, and describe the programming model in more detail. We also include experiments comparing our solution to similar runtime systems and show that our solution has less overhead than all the other evaluated systems. Our implementation is open source and freely available at GitHub. ${ }^{1}$

1.4. Related work. The question of how to efficiently take advantage of multicore architectures has been investigated in the setting of linear algebra, where performance is key. It has been concluded that task-based, dynamically scheduled approaches are well suited for taking advantage of multicore processors $[15,6]$.

There are several different task-based programming models. The main difference between them is how dependencies are managed. Either all tasks can execute immediately after they are spawned, or there is some way to specify dependencies that must be satisfied before the tasks can execute.

One of the best known task-based programming languages is Cilk [5] (now Intel Cilk Plus [13]). This is a language extension to C (and to C ++ in Cilk $++[18]$ and Intel Cilk Plus) which introduces the spawn keyword to generate new tasks, which are immediately allowed to run, and the sync keyword, which blocks until all tasks spawned from the current task are finished. This provides the fork-join programming model and may cause unnecessary synchronization.

The most common programming model for shared memory parallelism is OpenMP. Tasks were introduced into the OpenMP standard with OpenMP 3.0 [2], following the same task model as Cilk. Sections annotated with \#pragma omp task are spawned as tasks and are immediately allowed to run while the control flow continues after the task. To synchronize tasks, there is the \#pragma omp taskwait directive, which blocks until all tasks spawned by the current task are finished. OpenMP also provides parallel sections and parallel for-loops. Common for these constructs is that they provide the fork-join model. In OpenMP 4.0 [20], more general dependencies have been introduced through the depend clause.

The shortcomings of the fork-join model have been pointed out earlier in the context of efficient linear algebra algorithms for multicore systems [15]. There, it was shown that the fork-join structure arising from calling parallel subroutines from a sequential algorithm limits scalability. By instead implementing the algorithm as an explicitly parallel code that called sequential subroutines, the number of synchronization points was reduced, and the performance was improved. In the same work,

\footnotetext{
${ }^{1}$ https://github.com/tillenius/superglue
} 
different orders of executing the computations were considered, and it was concluded that the order of the computations should be decided at runtime for best performance.

To manage high degrees of thread level parallelism, it has been concluded in earlier work that algorithms must have fine granularity and be asynchronous. Operations should be split into tasks that work on data sets small enough to fit in the cache, and these tasks should be scheduled dynamically based on dependencies between them [6].

Intel Threading Building Blocks (Intel TBB) [14] is a C++ library for shared memory parallelism. It includes a task scheduler which besides the fork-join model allows dependencies between tasks to be specified explicitly and arbitrary DAGs of tasks to be built.

A problem with explicitly building a task dependency graph is that it may be demanding for the programmer. In order to realize all dependencies, the programmer must know all data that a task reads and writes since this will create dependencies. When dependency graphs are explicitly built, the programmer must also know what other tasks access the same data and declare dependencies between these tasks. This makes building the graphs difficult and error-prone. An improvement of this is to let the programmer annotate each task with which data it accesses. The problem of keeping track of which other tasks access the same data is then left to a runtime system, and the programmer is relieved of this responsibility.

A programming model where task dependencies are deduced from data accesses is the StarSs model. This programming model was introduced in CellSs [4], a system that targets the Cell BE processor architecture, and was applied for general multicore architectures in the SMP Superscalar (SMPSs) system [21]. The StarSs programming model is now represented by OmpSs [9], which succeeds SMPSs and adds support for heterogeneous architectures. In the StarSs programming model, the task declaration clause in OpenMP is extended with in, out, and inout clauses for declaring data dependencies. Using these clauses, the programmer specifies which data each task accesses and how the data is accessed (read, write, or both). OmpSs then uses this information to build a task dependency graph at runtime. The idea to deduce task dependencies from annotations of data accesses is not unique for the StarSs model but has been used earlier in, for instance, the Jade programming language [17].

StarPU [1] is a C library for task-based programming, targeting heterogeneous architectures. It is capable of scheduling tasks over (for instance) both CPUs and GPUs. It also manages data transfers and supports distributed memory parallelism. StarPU provides both the possibility to state dependencies between tasks explicitly and to state them implicitly through data dependencies. StarPU also includes a GCC plugin, which allows tasks to be annotated using pragma directives.

XKaapi [11] is a C++ library for task-based programming, with dependencies computed at runtime from memory access annotations. The programming model is borrowed from its successor Athapascan-1 [10]. XKaapi includes a compiler that introduces pragma directives which can be used for specifying tasks, as an alternative to the $\mathrm{C}++$ interface.

The Swan programming language [27] is an extension of Cilk which adds the possibility to annotate tasks with data access information, in order to introduce task dependencies. Swan uses an independently developed versioning system similar to the scheme we introduce here.

The dependency-aware task-based model is well suited for high performance computing. The state of the art in linear algebra on multicore architectures is the PLASMA package [8], which uses a C library for task-based programming with data 
dependencies called Quark [28].

1.5. Paper organization. The next section introduces our programming model, and section 3 covers the details. We highlight the flexibility of our solution by showing features, usage examples, and showing how to customize the library to the user's needs in section 4 . To evaluate the performance of our implementation, we have conducted a number of experiments: We show results from microbenchmarks in section 5 , present more realistic applications in section 6 , and show comparisons against other task-based frameworks in section 7 and against highly optimized third-party code in section 8 .

2. Our programming model. Our programming model contains two fundamental concepts: handles and tasks. Handles are objects used for synchronization. They represent some shared resource for which accesses should be managed. The most common shared resource is data, but handles can be used to represent anything. A handle may represent network communication or file access and can be used to serialize or to order the use of such resources.

The programmer creates handles to protect shared data and writes tasks which operate on this data to perform the logic of the software. The software is then expressed by creating and submitting these tasks to the runtime system. For each task, the programmer must specify which handles are required and what type of access the task performs, such as whether the tasks only read or also modify the protected data. From the order the tasks are submitted, or rather from the order the handles are accessed, together with the access type, the runtime system deduces which tasks can be executed in parallel and maps the tasks to the available cores in the system.

2.1. Handles. One way to think of handles is that they are similar to ordinary locks in the way that it is up to the programmer to decide what they protect and that their meaning is unknown to the runtime system. The programmer must correctly specify all handles a task accesses, or the program will be incorrect. The runtime system cannot help with this.

In contrast to programming with locks, it is not possible to have deadlocks. Instead of blocking and waiting for a handle to become available, tasks are queued at a handle, while the runtime system executes other tasks instead.

In our model, accesses are registered to handles, not to memory addresses. There are several reasons for this choice. First, the memory address is not necessarily a unique identifier. Different tasks can have pointers to the same memory block but update different parts of the block. An example of this that has been reported earlier is when several tasks of a matrix decomposition algorithm access the same matrix block but some only touch the lower triangular part and others only access the upper triangular part [16]. Another reason is that when working with memory addresses, the user could expect the runtime system to detect that synchronization is needed between tasks that access the same data through different pointers. This could also be supported, but at an additional cost at runtime. With handles, it is clear that the library cannot find out whether two handles represent overlapping resources. A third reason is that the handles keep the required book-keeping data, and by requiring the user to provide the handles directly, we do not have to map the address to a bookkeeping object. The possibility of using memory addresses to represent data and then mapping addresses to book-keeping objects is still available and can be added as a layer upon our solution.

The granularity of the handles is connected to the granularity of the tasks and is 
an important factor for performance. If tasks are too large, or similarly, handles too coarse, there will be fewer tasks to run in parallel and less parallelism. If tasks are too small, too much time will instead be spent on task and dependency management. The granularity of the tasks depends strongly on the granularity of the handles. There needs to be enough handles for tasks to work on in parallel, but too many handles occupies more memory and leads to too small tasks.

Tasks should be large enough to make the time spent on task management negligible. Later we perform experiments with different task sizes, giving a hint of how large tasks need to be. SuperGlue does not help the programmer make these decisions.

The lifetime of a handle naturally needs to be longer than all tasks that access it. Handles can be created dynamically as needed and can be deleted either when it is certain that all tasks that access it have finished, such as after a global barrier, in a task that does not access the handle but is guaranteed to execute after the last task that does, or in the destructor of the last task that accesses the handle.

2.2. Tasks. A task is a piece of the program logic together with the data needed for its execution. In practice, it is an object that inherits a Task class provided by SuperGlue, with a callback method that is called to execute the task. When a task is constructed, the data that is required for the task, typically pointers to the data it works on, are stored in the task object, and accesses are registered to the required handles. Before a task is submitted to the runtime system, the programmer must specify which handles the task accesses, together with the type of each access. This information is stored in the task and is used by the runtime system to deduce whether the task is ready to execute. Tasks are executed as a whole and cannot be suspended. They are therefore expected not to block.

2.3. Access types. To allow concurrent access when desired and unique access when required, the programmer must specify an access type for each handle a task accesses. The default access types are read, write, and a type for reductions called add, but it is possible to customize these, as will be described in section 4 .

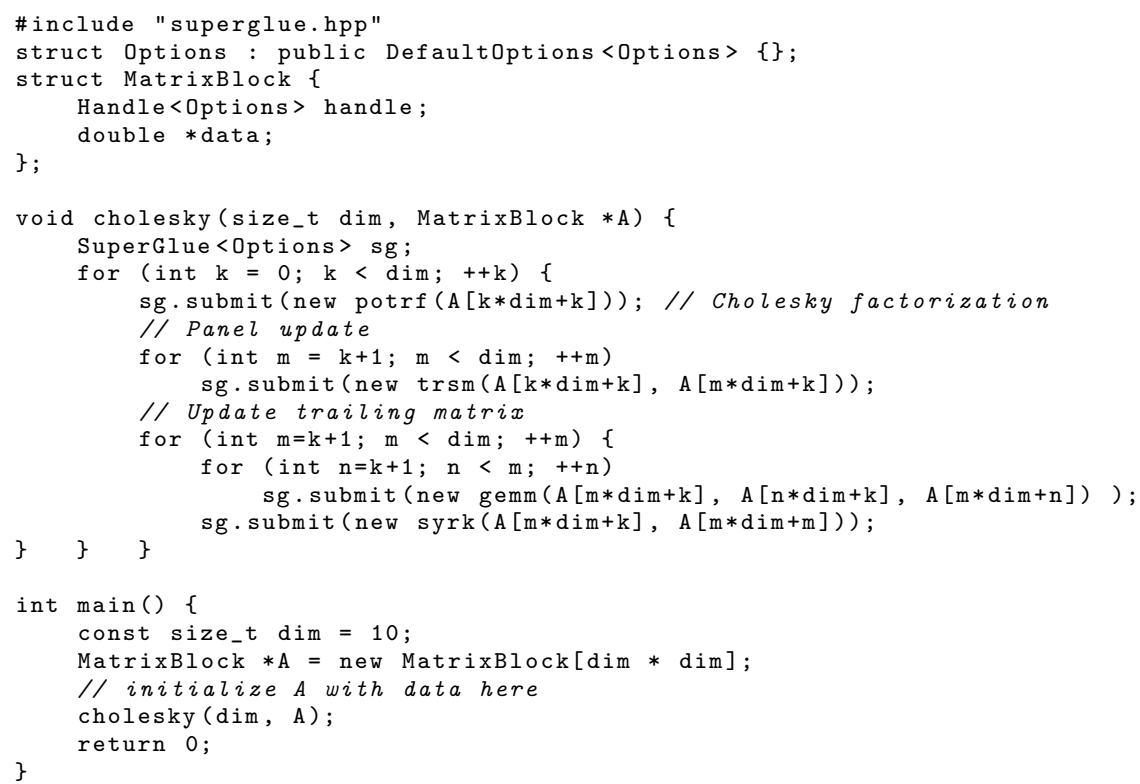


The read access type means that the task must wait for all previous write or add accesses to finish but that several tasks may read the same handle concurrently.

A write access means that the task must wait for all previous accesses to finish before it can execute. The task is then allowed to both read and write to the data. There is no access type to describe a write-only access.

The add access type means that a task must wait for all previous read and write accesses to finish. Two tasks with add accesses to the same handle can execute in any order but not concurrently. The typical use for this type is to accumulate results together. The type is not restricted to addition but can be used for any tasks that perform associative and commutative operations, or other operations where exclusive access is required, but order is not important. The name add is chosen because it is a common case and a simple name.

2.4. Example. Listing 1 shows an example of an application that uses SuperGlue. It performs a tiled Cholesky factorization to decompose a symmetric positive definite matrix $A$ into a lower triangular matrix $L$ such that $A=L L^{T}$. The tiled Cholesky factorization is a classic example of an algorithm with nontrivial dependencies, but the details of the algorithm are not important here. By creating a SuperGlue object, as on line 9, the SuperGlue runtime system is initialized, and worker threads are created so that there is one thread per available processor core. SuperGlue can be configured to meet the application needs, as will be explained in section 4 . This configuration is done by specifying types in an Options struct, which most other SuperGlue classes take as a template parameter. The Options struct is defined in line 2, where it in this case only inherits and uses the default options.

The algorithm works on tiles of a large matrix. To manage dependencies between the different calls to BLAS kernels, a handle is associated with each matrix tile. As shown in line 3, a handle has been added to the struct that represents a tile of the matrix. Tasks are submitted to the runtime system using the submit method, as shown, for instance, in line 11.

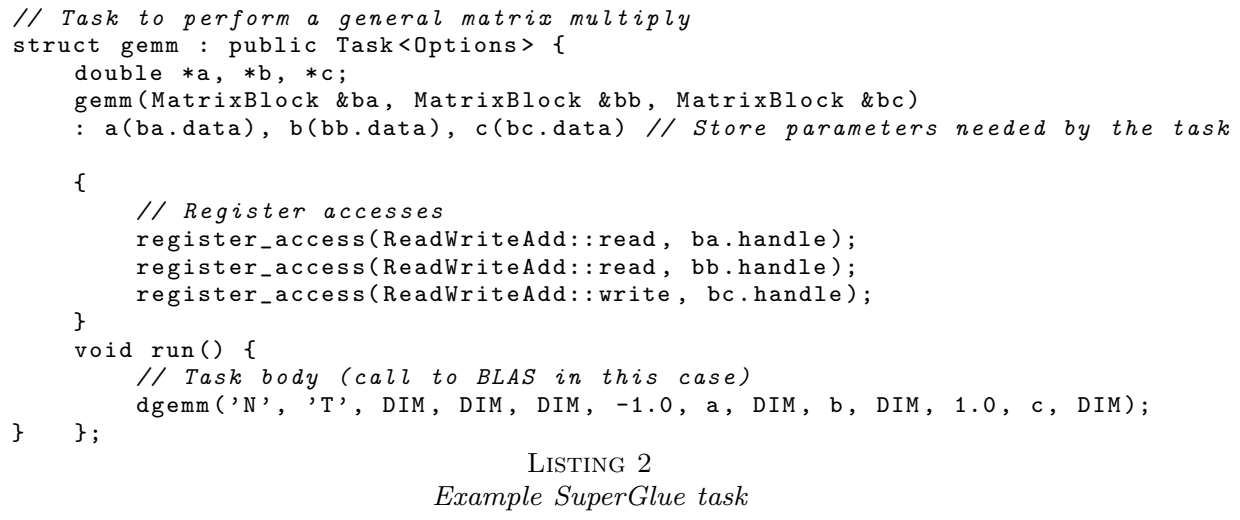

LISTING 2

Example SuperGlue task

An example task definition is shown in Listing 2. This task performs a matrix-matrix multiplication by calling BLAS. It takes two input matrix tiles ba and bb and adds their product into the output matrix tile bc. A task in SuperGlue inherits from a Task class, as in line 2. The constructor (lines 4-11) copies the data needed for the task into the class (line 5) and declares which handles the task accesses. The tasks read from ba and bb, so read accesses are registered on line 8 and 9 , and a write access to $\mathrm{bc}$ is registered on line 10 . 


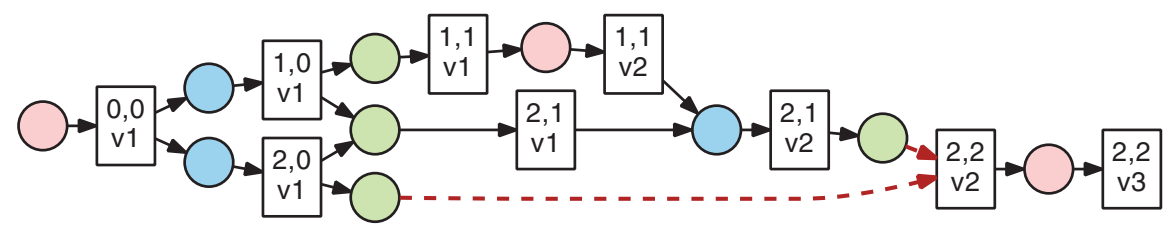

Fig. 2. Data dependency graph. Circles are tasks, edges are dependencies, and boxes are handles. The same handle is represented by several boxes, one for each version. The first line in each box is the name of a handle, and the second line is the handle version. The dashed edges mean that tasks can access the handle in any order but not concurrently.

When all the dependencies of the task are met, the runtime system will call the run method of the task (line 12), which will invoke BLAS to perform the actual matrix multiplication.

2.5. Data-centric view. Central to this programming model is the data-centric view. In this model, tasks can depend only on data, not on other tasks. One way to illustrate dependencies in this data-centric view is shown in Figure 2. This figure shows the same dependencies as Figure 1 but also includes the handles.

This view simplifies the implementation. The straightforward way for a runtime system to insert a new task into a dependency graph using information about which memory addresses the task accesses is as follows:

1. Look up the memory address in a map to find the book-keeping object.

2. Lock the book-keeping object to avoid data races.

3. Check which preceding tasks to wait for from the book-keeping object.

4. For each preceding task:

Lock the preceding task, and add a dependency edge to it.

5. Update the book-keeping object with the access from the new task.

In our model, we avoid looking up the book-keeping object and instead require that the user provide the correct handle. By having tasks depend on data instead of on other tasks, we avoid synchronization between tasks, which are transient in nature. Data objects that are shared between the tasks are required to be alive until all tasks are finished, while tasks can be submitted, executed, and deleted at any point. To submit a new task in our model, the following steps are needed:

1. Lock the handle to avoid data races.

2. Update the handle with the access from the new task.

The actual update is simple. All that needs to be done is to increment one or two integer variables, depending on the access type.

Not only does the data-centric view simplify implementation, but it also improves flexibility. In our model, it is possible to register future accesses to a handle. This way it is possible to submit a task that waits for a task that will be submitted some time in the future, in a natural and straightforward way.

3. The runtime system. The runtime system has one worker thread per core, and each worker thread has its own queue of ready tasks. The worker threads execute tasks from the front of the ready queue. If a task submits new tasks, they are put in the front of the ready queue of the thread where the task is running. If a worker runs out of tasks, it tries to steal tasks the from the end of another thread's ready queue, picked at random.

In addition to the ready queues at each worker, there is also a task queue at each handle for tasks that have an unfulfilled dependency on that handle. This leads to a 


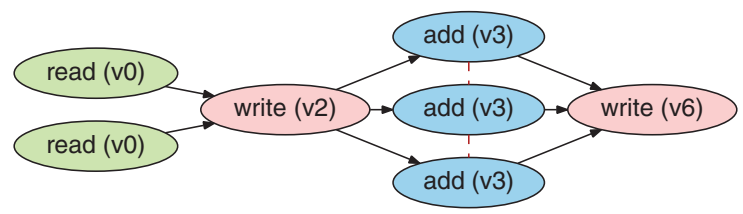

Fig. 3. Example illustrating how dependencies are represented by versioning. Nodes are tasks, the label and color (seen online only) specify the access type, and the required version number is given in the parentheses. All tasks access the same handle. The dashed lines between the add tasks denote that they must not run at the same time.

large number of task queues and reduces contention on task queues to a minimum.

3.1. Dependency management. Dependencies are managed by attaching a version number to each handle and having the tasks require certain versions of the handles it accesses. The version number works as a counter that keeps track of how many tasks have accessed the handle and is increased after each access to it. Note that the version number is increased also after read-only accesses. This is needed to detect whether all previous reads are finished, before a write can be allowed.

When a task is submitted to the runtime system, it will be assigned a required version number for each handle, which determines when it will be able to execute. The required version of a handle is available when its current version is equal to or greater than the required version. A task is ready to execute when the required versions of all handles it accesses are available.

If a task requires a version of a handle that is not yet available, the task will be put in a queue at that handle. When a worker thread has finished a task, it will increase the versions of the accessed handles and wake up the tasks that waited for the newly available version.

Figure 3 illustrates how dependencies are represented. In this example, all tasks access a single handle. The two first tasks only read from the handle and can run at the same time. They will both require version 0 of the handle. The next task is a write, and it must wait for the two previous read tasks to finish, so it will require version 2 of the handle. This write is followed by three add tasks, which can run in any order but not at the same time. They will all require version 3, which means they have to wait for all three previously submitted tasks that access this handle to finish. The last task is a write and must wait for all previously submitted tasks to finish, and thus it requires version 6 of the data. It is worth pointing out that we do not keep several copies of the data that a handle represents. This might be desirable in some cases and is discussed further in section 4.

The dependency checking is easily implemented. To see whether a task is ready to execute, it is enough to compare the required version with the version of the handle. Which version a task should require depends on the access type. If the previous access was of the same access type, and that type allows reordering or concurrent execution, the same version is required. Otherwise, the required version is one more than the total number of accesses registered so far.

It is enough to save the access type, the current version, and the total number of accesses for this book-keeping. For the default configuration, with read, write, and add as the possible access types, it suffices to store only two versions: the version to require if the next access is a read, and the version to require if the next access is an add. Write accesses need to wait for all previous read and add accesses to finish, and 
moved into a ready queue. Once a task is moved into a ready queue it is guaranteed to be ready to run, and no more checks are needed. From a ready queue it might be stolen at most once since a successfully stolen task is executed directly. When add accesses are used, there is no guaranteed limit on the number of tries required before a task successfully acquires exclusive access to a handle.

There are no global scheduling decisions or global data structures in this scheme. Tasks that are ready to execute when they are submitted will be distributed among the threads, unless they are submitted from within a task directly to the ready queue of the current thread. Except for that, communication between threads only happens when load balancing is needed (stealing), when tasks on different threads access the same data, or when there is a global barrier.

4. Customization and features. SuperGlue is written as a research tool for experimenting with task-based models. Because of this, several classes can be overridden to change the default behavior, and features can be enabled or disabled to experiment with different solutions. Configuration is done by defining types in an Options struct, which is given as a template parameter to all SuperGlue classes. This means that disabled features are disabled at compile time and cause no memory or runtime overhead. This section is intended to give an idea of what can be customized, and how, by showing a few examples, but the list is not complete and the details are suppressed.

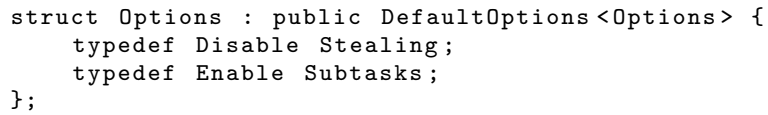

Example Options struct. This example disables task stealing and enables the possibility for a task to create subtasks and wait for all subtasks to finish before the task is finished.

Listing 6 shows an example Options struct. This struct is used as a template parameter to all SuperGlue classes, which allows settings to be made at compile time. The default options class defines the types Enable and Disable, which are used to indicate that features should be enabled or disabled. In this example, task stealing is disabled, and the Subtasks feature is activated. The Subtasks feature enables a task to submit subtasks and wait for all subtasks to finish before the task itself finishes.

4.1. Scheduling. It is possible to override the default behavior to affect the scheduling in three places:

- which task to select for execution from the local ready queue,

- which task to steal from the ready queue of another thread, and

- which other task queue to try to steal tasks from.

The default behavior is to pick the first task of the local ready queue and to steal the last task from a foreign queue. This can be customized by overriding the default task queue with a custom one where the pop_front and pop_back operations select tasks using the desired criteria. The task queue class can also define additional data fields (possibly none) that should be included in the task class. This is used to attach a priority field to all tasks or, for example, to implement intrusive linked lists. Which task queue implementation to use is decided by the ReadyListType type in the Options struct. By redefining this type, a user-supplied task queue can be used instead.

The default strategy for picking a queue to steal from is to pick a queue at random, and then attempt to steal from the queues in order, starting from that one, until a task is successfully stolen, or all task queues have been attempted. This behavior can 
be customized by implementing a class that defines a steal method that attempts to steal tasks from other task queues and redefining the StealOrder type in the Options struct to use this new implementation instead of the default one.

Related to scheduling is setting thread affinity. The default behavior is to pin each thread to its own core in the order the cores are enumerated by the operating system. This can be overridden to implement a different pinning strategy, or to disable pinning entirely, by writing a new class and to redefine the type ThreadAffinity in the Options struct.

4.2. Memory management. Memory management is avoided in SuperGlue whenever possible and is only done in two places: to free tasks that have finished, and in the container used in handles to keep tasks waiting for future versions. The container uses a $\mathrm{C}++$ standard library allocator, whose type is defined in the Options struct. To free memory used for storing tasks, the Options struct contains a FreeTask type, that has to provide a free () method, which will be called with the finished task as a parameter. By default, SuperGlue uses the default $\mathrm{C}++$ allocator. This choice avoids external dependencies, but the default allocator is known to be a potential scalability bottleneck. By overriding these types, the allocator can be replaced with a custom allocator that scales better or is better suited in some other way.

4.3. User-defined access types. It is possible for SuperGlue users to use a different set of access types than the default ones. When defining an access type, the programmer needs to declare whether it can be reordered with other accesses of the same type and, if it can, also whether the accesses need to be exclusive or not.

As an example, if some tasks perform an additive operation, while other tasks perform a multiplicative operation, tasks performing the same access types can be reordered, while order must be preserved among tasks with different access types. One might also want to add an access type that allows several tasks to modify data concurrently. This allows data races or lets the user manage data races manually.

4.4. Renaming. There is no write-only access type in SuperGlue. A writeonly access would signal that a new instance of the data can be created, and a task could start working on it immediately, without having to wait for previous tasks that need the old data to finish first. This avoids a write-after-read dependency and is called renaming for its similarities with register renaming performed in processors. SuperGlue does not support automatic renaming since it has no control over the memory buffers that the handles represent. Also, automatic renaming is not always desired. If there already is enough parallelism, it is better to let write-only tasks wait and reuse the same buffer. If the runtime system creates new buffers automatically, there is both a risk of completely running out of memory and a risk of performance loss due to a larger working set and more cache misses.

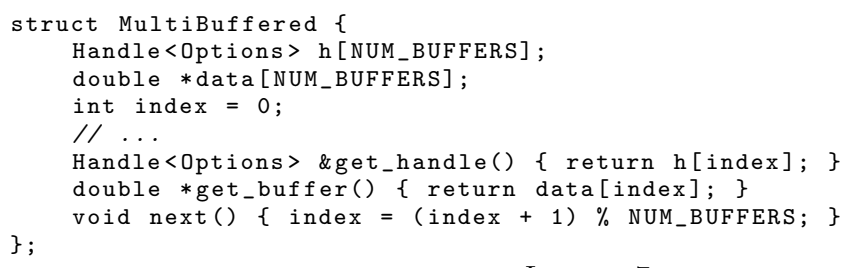

LISTING 7

Example of using multiple buffering to avoid write-after-read dependencies. This implementation is not thread safe, but works if all tasks are submitted from a single thread, and can easily be extended to be thread safe. 
One way to avoid write-after-read dependencies is to use multiple buffering explicitly. Listing 7 illustrates the idea. Each buffer gets its own handle, and write-only operations call the next method before registering their write access. Note that this implementation has the drawback that it always alternate buffers, even if all tasks that accessed the old buffer have already finished.

4.5. Parallel reduction. There is some support for automatic renaming for add accesses, in order to allow parallel reductions. When this feature is enabled, a task that needs exclusive access to a handle that is locked will still be able to execute. The task must then write its partial results into another buffer instead. When the task is finished, it attaches this buffer to the handle. The handle can only keep a single reduction buffer. If a buffer is already attached when a task wants to attach one, it is detached and merged with the new buffer using a user-defined function. The process is then repeated to attach the merged buffer to the handle.

This allows tasks that use the add access to run in parallel. The partial results are merged lazily and are merged in parallel if several threads attach partial results concurrently. The buffers are not guaranteed to be merged until a task accesses the handle with a different access type.

The user must provide functions for creating new buffers and initializing them, merging two buffers, and applying a buffer to the real destination. Since the merge and the apply operations are two separate functions, it is possible to have different representations in the temporary buffers and in the real destination buffer. When a task is declared to support this feature, the user must program the tasks to check where the output should be written. The tasks must support three possibilities: write to the real destination, write to an existing temporary buffer, or create and initialize a new temporary buffer and write to this.

4.6. Thread workspace. For tasks that require temporary work space buffers, there is an option to enable each thread to preallocate a buffer of a determined size. Tasks can then request work space memory from this buffer, which is guaranteed to be local to the thread, and need not be allocated and freed for each task.

4.7. Visualization and instrumentation. A positive effect of the task-based model is that the beginning and end of each task execution are natural locations for instrumenting the software. By storing the start and end time of each task, and on which thread it was executed, a task execution trace as in Figure 4 can be drawn. This is a trace from executing a program with dependencies such as those illustrated in Figure 1(a) and Figure 2. From such an execution trace it is possible to see when tasks are started, for how long they execute, and on which thread they were executed. We use triangles instead of rectangles to illustrate tasks, as we find that it makes it easier to separate several small tasks from a large task and easier to detect idle time between tasks. From an execution trace, it is possible to directly see whether there is enough parallelism, whether any cores are idle, in which parts of the program most time is spent, and whether there is any unexpected behavior. We will use these execution traces to evaluate how well programs are scheduled. In [25], execution traces are used to detect tasks that exhibit performance degradation due to shared resources such as memory bandwidth. This information is then used to insert scheduling constraints that prevents contention-sensitive tasks from being executed concurrently.

Instrumentation is activated by defining a type in the Options struct with member functions that will be called before and after each task invocation. In addition to generating execution traces as in Figure 4, SuperGlue includes example instrumen- 


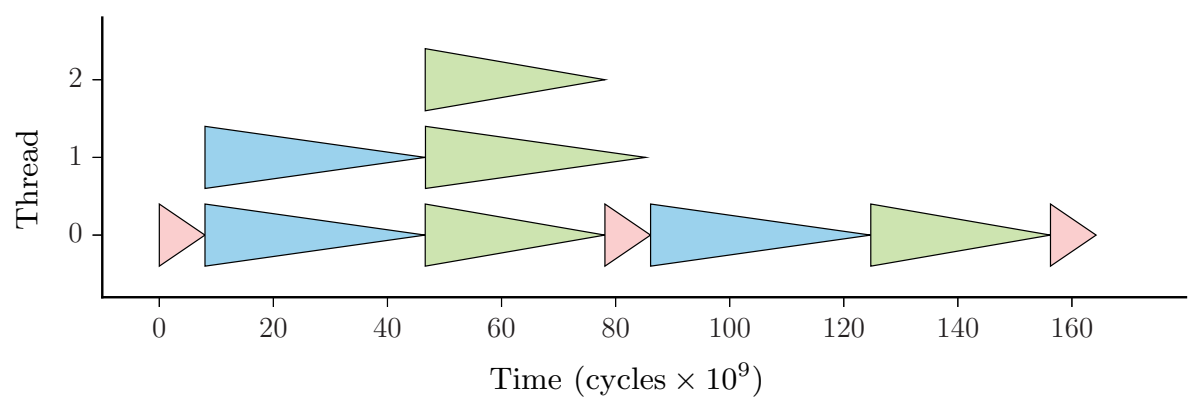

FIG. 4. An execution trace illustrating how a task-based application is executed. Each triangle represents a task, and colors distinguish different task types.

tation classes to read performance counters before and after each executed task, and there is also an option to generate task dependency graphs in Graphviz DOT format for illustrating the dependencies in an application. The SuperGlue source code repository contains two examples of how execution traces can be visualized: a $\mathrm{C}++$ application that uses OpenGL and glut, and a Python script that uses matplotlib.

When execution traces are recorded, each thread stores the time before and after each task is executed, together with a description string, in a vector in thread-local storage. The information is written to disk first when the application exits. Execution traces may require a large amount of memory and incur some overhead. To verify that the instrumentation does not disturb the execution, the application can be run once with instrumentation enabled and once with it disabled. If the total execution time is affected, the recorded data cannot be trusted. When tasks are large enough for the overhead from SuperGlue to be negligible, the additional overhead caused by enabling instrumentation is usually too small to be measured.

4.8. Interoperability. SuperGlue has a $\mathrm{C}$ interface to allow it to be used from $\mathrm{C}$ and other programming languages. An early version of this interface was used to use SuperGlue together with existing code written in Fortran [22], and it has also been used in a software for epidemiological simulation written in C [3]. The interface only exposes the core functionality of SuperGlue and does not support customization, but it can be used as a template for how to build a custom interface with settings other than the defaults.

5. Microbenchmarks. This section evaluates the performance of the proposed programming model by running experiments on our SuperGlue implementation.

Time is measured by reading the time stamp counter using the rdtsc instruction. This counter has a constant rate and behaves like a wall-clock timer. Each thread is pinned to its own core or hardware thread in all experiments. When not all cores are used, we first allocate one thread per module and per core, and only when all modules or cores are already occupied do we assign workers to share a module or a core.

There are no parameters in the SuperGlue implementation that are tuned for the specific hardware used in the experiments.

5.1. Scaling with respect to number of cores. To ensure that our solution scales well to large numbers of cores, we have performed tests on a Xeon Phi 5110P, with 60 cores and 240 hardware threads. The experiment computed a tiled Cholesky factorization of a matrix of size $16384 \times 16384$, divided into tiles of $256 \times 256$ elements. 


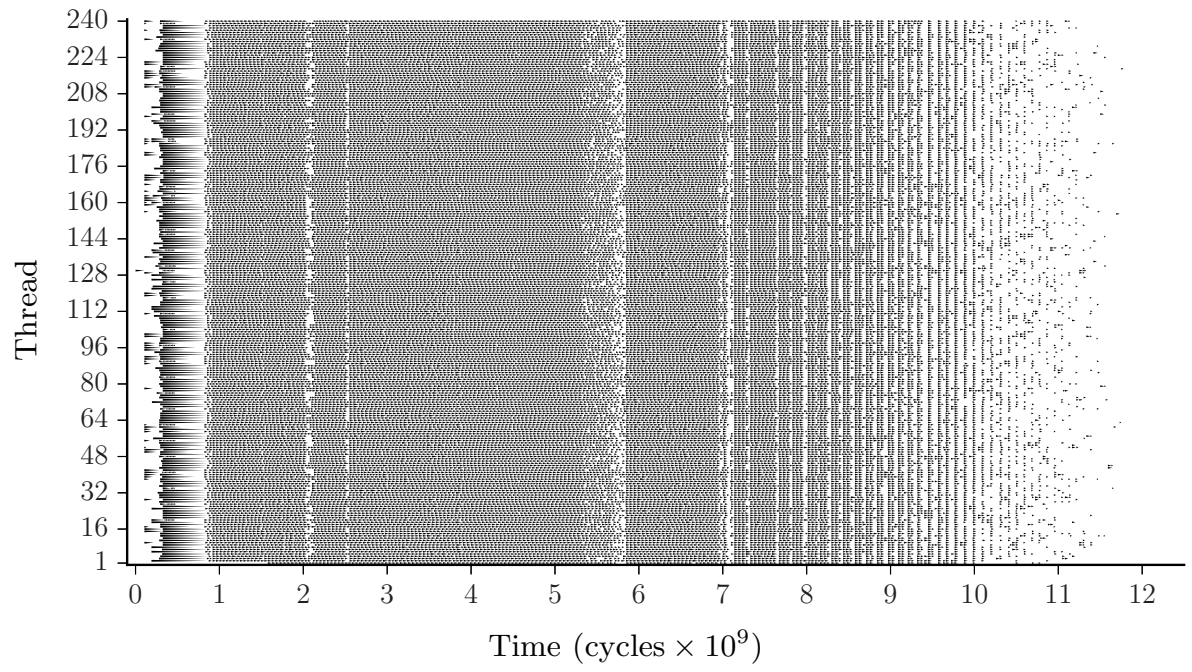

FIG. 5. Execution trace of a Cholesky factorization run on the 240 hardware threads of a 60-core Intel Xeon Phi. The matrix size is $16384 \times 16384$, divided into $64 \times 64$ tiles of $256 \times 256$ elements each.

The execution trace is shown in Figure 5. In the execution trace, it can be seen that some time is needed to start up and reach full speed, which is not reached until at about time 1. In the end of the execution trace, there is less parallelism in the algorithm, which leads to tasks being executed more sparsely. Just after time 2, before time 6 , and at time 7 , there are short intervals where the application runs out of tasks to execute and worker threads are idle. The reason for these imperfections is not clear, but suboptimal scheduling cannot be ruled out. Introducing task priorities and prioritizing tasks on the critical path, which in this case are the tasks that work on the diagonal tiles, did not improve on this behavior.

In this experiment, only $131 \mathrm{GFlop} / \mathrm{s}$ was reached, while the theoretical peak is 1011 GFlop $/$ s. $^{2}$ The kernels used in this experiment only reached 2.35 GFlop/s when running on a single thread. The parallel version was $55.8 \times$ faster, close to the ideal $60 \times$. The purpose of this experiment is to verify that the runtime system scales up to large numbers of cores. After the start-up effects and before the algorithm runs out of parallelism, the execution trace shows that tasks are scheduled densely and as desired and that SuperGlue successfully scales up to at least 240 threads.

5.2. Scaling with respect to task size. To investigate how well our solution scales for different task granularities, we measure the time it takes to execute a number of identical and independent tasks that perform no work but only wait for a number of cycles. The tasks perform no work in order to isolate the behavior of the runtime system and to have higher precision in the control of the granularity. This means that we investigate the ideal case when tasks do not affect other running tasks by competing for shared resources such as cache or memory bandwidth. The time is measured from when the first task starts until the last task finishes and does not include time for startup or shutdown.

\footnotetext{
${ }^{2}$ The theoretical peak was calculated as 8 double wide vector registers $\times 2$ operations per instruction with fused multiply and add $\times 60$ cores $\times 1052.63 \mathrm{MHz}$.
} 


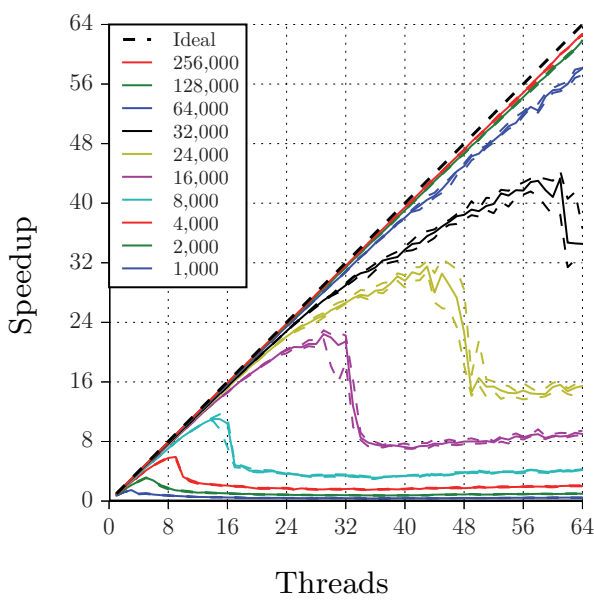

(a) Bulldozer

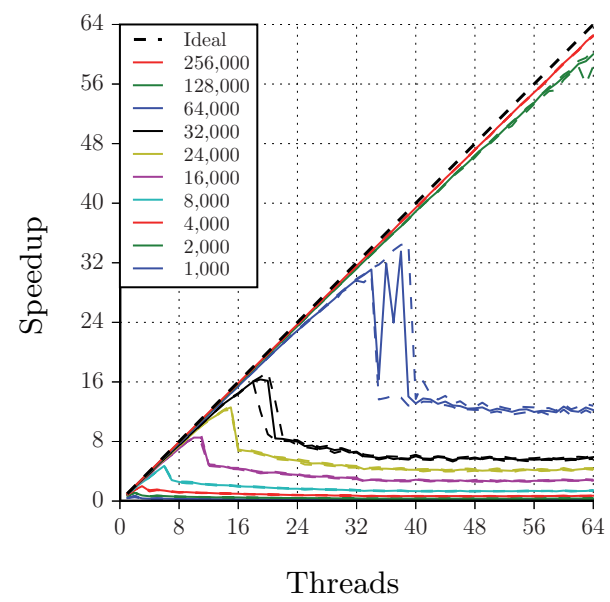

(b) Sandy Bridge

FIG. 6. Scaling with respect to task size, where task size is the execution time of a single task measured in cycles. The graph shows the speedup over the ideal execution time (number of tasks $\times$ task size / threads). Each line corresponds to a task size. The solid lines show the median, and dashed lines show the upper and lower quartiles of 15 runs. The experiment is performed on two different computer architectures: Bulldozer and Sandy Bridge.

Memory management of tasks impacts the performance. To make the experiment less dependent on the memory allocator, all memory to store tasks is allocated in advance and returned first after all tasks are executed, so that no memory management of the tasks is included in the timing. This experiment is repeated in section 7 , where we compare our solution to other projects, with standard memory allocation included in the timing.

The experiment is conducted on two different computer systems. The first is called Bulldozer and has four AMD Opteron 6276 processors based on the Bulldozer architecture. Each processor has eight modules, and each module contains two cores and a single floating point unit that is shared between the two cores. This results in 64 cores running at $2.3 \mathrm{GHz}$. The second is called Sandy Bridge and has four Intel Xeon E5-4650 based on the Sandy Bridge architecture, giving a total of 32 cores running at $2.7 \mathrm{GHz}$. The tests were run with hyperthreading enabled, which results in 64 hardware threads.

The number of tasks is selected to 6,400 , which is large enough to fill all the threads and large enough to make the steady state dominant and the irregular behavior at startup and shutdown less significant. Increasing the number of tasks did not affect the results in a qualitative way. The experiment is repeated 15 times for each task granularity to capture dispersion in execution time.

Figure 6 shows how large tasks must be to reach good speedup and what happens when tasks are too small. The figure shows speedup over the ideal execution time on one thread, where the ideal execution time is the number of tasks times the number of cycles per task. Note that it is impossible to reach perfect efficiency since that would require every single clock cycle to be spent inside a task and leave no cycles for task management.

On Bulldozer, good scaling is reached when tasks are at least 64,000 cycles, cor- 


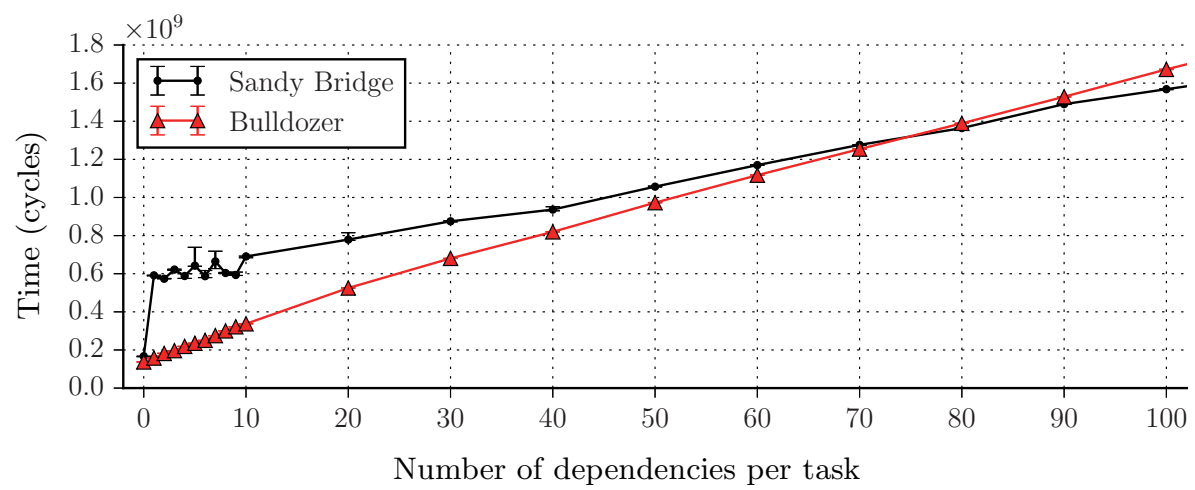

FIG. 7. Scaling with respect to number of dependencies. 64,000 tasks executed on 64 cores, each waiting for 128, 000 cycles, and accessing a varying number of handles. Error bars show the first and third quartiles of 15 runs but are barely visible due to low dispersion.

responding to just below $30 \mu \mathrm{s}$. On Sandy Bridge, tasks should take at least 128,000 cycles, corresponding to just below $50 \mu$ s on that architecture. The Sandy Bridge machine has 32 cores, but since the tasks only read the time stamp counter, it is possible to scale up to almost 64 times speedup using hyperthreads. When actual work is performed, the maximal speedup is expected to be 32 .

Increasing the number of threads when the task size is too small can lead to a sharp drop in performance. This is due to contention on the locks protecting the task queues. These locks are simple test-and-test-and-set spinlocks with no backoff. We have performed experiments with more advanced locks, but the benefits did not outweigh the increased cost of acquiring or releasing the locks, at least in our implementations. Adding a back-off helps against the sharp drop in performance, but it is unclear how to select good parameters that work for all platforms. Since it would be unfair to tune the locks for the specific machines that we use to perform our experiments on when we compare against other systems in section 7 , we opted to use the simple and parameter free spinlocks.

5.3. Scaling with respect to number of dependencies. The aim of this benchmark is to measure how overhead for dependency management scales with number of dependencies. 64,000 tasks are submitted, which is a large enough number to make the long-term behavior dominant and startup and shutdown anomalies negligible. Each task waits for 128,000 cycles, selected to be long enough to avoid contention according to the results from section 5.2. Each test was repeated 15 times, in order to capture dispersion in execution times. Memory is allocated in advance and is not freed until after the experiment. All tasks are submitted before any task is allowed to execute, and both task submission and execution are included in the timing.

The experiment was run on both the Sandy Bridge and the Bulldozer machine, and the results are shown in Figure 7. On Bulldozer, the execution time is close to linear in the number of dependencies, but the slope changes slightly from about 300 cycles per dependency to 250 cycles per dependency. On Sandy Bridge, there is a larger cost for introducing dependencies, which we have no explanation for. For large number of dependencies, the overhead approaches 217 cycles per dependency.

We executed the test with up to 10,000 dependencies on Bulldozer, at which point all 64,000 tasks each must keep a list of its 10,000 dependencies, meaning that 640 


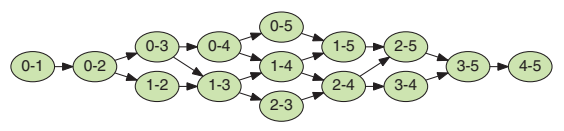

(a) Write accesses

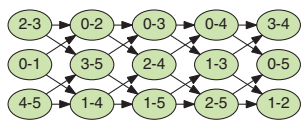

(b) Add accesses

FIG. 8. Comparison of the dependency graphs arising from using write (respectively, add) accesses for updates that can be reordered.

million items are stored in the memory. This is more than is practically useful. The mean overhead when each task had 10,000 dependencies was about 214 cycles per dependency.

From these tests we verify that our solution scales linearly with the number of dependencies and that it does not collapse when the number of dependencies is large. The results varied between the two machines but can be summarized as that there might be an initial cost to use dependencies, which was measured to about 6500 cycles on Sandy Bridge but was not seen on Bulldozer. When dependencies are used, the overhead to expect from adding another dependency was measured to between 200 and 300 cycles.

6. Applications. In order to show the usability of SuperGlue for real applications, this section contains a couple of more realistic applications that we have parallelized using SuperGlue. In addition to the applications presented here, SuperGlue has also been used in an implementation of the fast multipole method for hybrid CPU and GPU systems [12] and in an application for simulating how infectious diseases spread [3].

6.1. Direct $\boldsymbol{n}$-body simulation. The first application is an $n$-body simulation code that simulates how $n$ neutral atoms or molecules interact with each other, modeled by the Lennard-Jones potential. The simulation consists of two steps: calculating all the forces that act upon each particle, and moving the particles according to the forces acting upon them. The forces are calculated by the direct sum of all pairwise interactions. This method does not scale to large numbers of particles, but it is the computational kernel needed also in more efficient methods, such as the fast multipole method or Barnes-Hut.

The application is interesting because during the force calculations there are two tasks that update the force acting upon each particle. If these tasks are submitted in a straightforward order and the tasks are registered to write to the force field of the particles, the tasks are constrained to perform the updates in the order the tasks were submitted, which may allow very little parallelism. This is a known problem that has been studied before [19].

When the force field updates are registered as add accesses, tasks can be reordered and more parallelism can be extracted. This effect is illustrated in Figure 8. Here, numbers denote blocks of the force data. Two tasks may not update the same block at the same time, meaning that any two tasks that have a number in common have a dependency between them. Figure 8(a) shows the case when accesses are registered as writes and cannot be reordered, while Figure 8 (b) shows a possible dependency graph with more parallelism, arising from registering the updates as adds, which allows tasks to be reordered.

In this application, we used the renaming feature of SuperGlue. We simulated 8,192 particles grouped into 32 blocks of 256 particles each. The problem size and 
the number of blocks are on the limit of what is enough work for 64 threads and were selected to show how SuperGlue behaves when parallelism is scarce. Most force calculation tasks (taking over $95 \%$ of the execution time) write force contributions to two blocks of particles at once, which means that only 16 such tasks can run at the same time. One way around this problem is to create smaller blocks, but this would also increase the overhead. By using the renaming feature as described in section 4.4, force calculation tasks can run even if the output buffer is used by another task, in which case the results are written into a temporary buffer. Any such temporary buffers are then merged into the original buffer before it is read the next time.

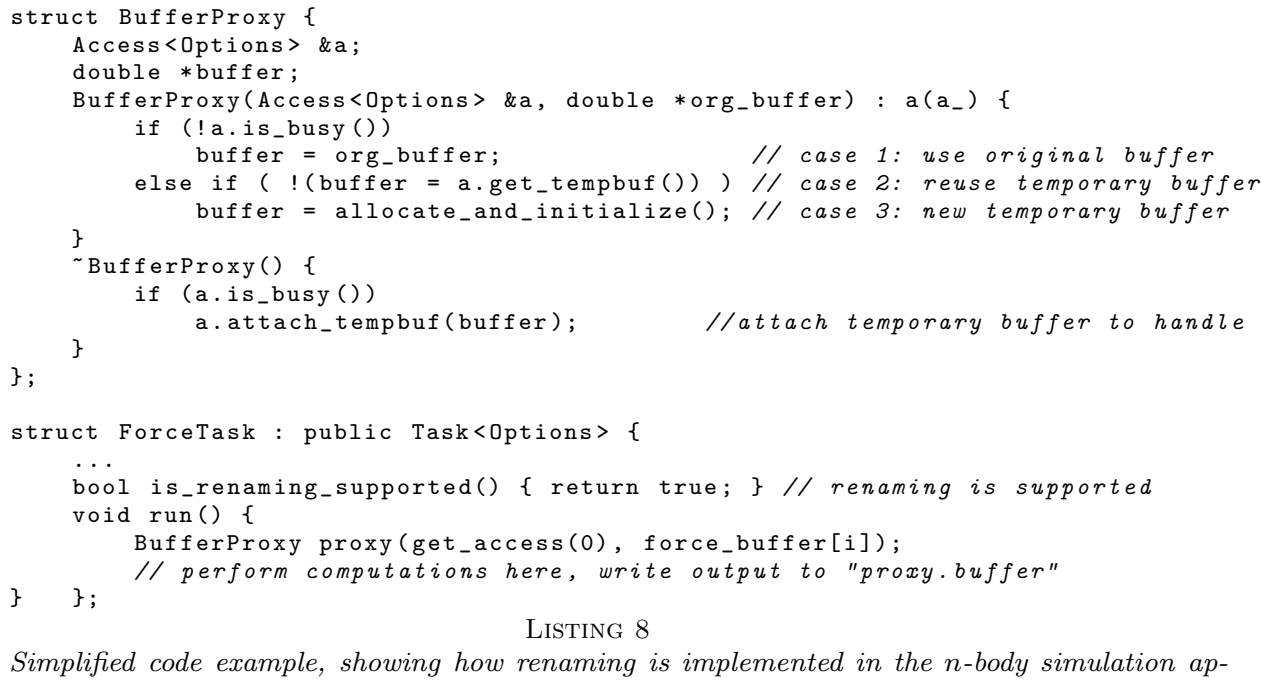

Listing 8 shows a slightly simplified version of how renaming is implemented in the $n$-body simulation. The Access class keeps a handle and the required version and can be retrieved from a task using the get_access() method. The user must implement an is_renaming_supported() method that returns true to indicate that SuperGlue can start tasks even when exclusive access could not be granted to all handles. To find out whether exclusive access was acquired for a handle, the Access class has an is_busy() method that returns true if renaming is required for its handle.

Figure 9 shows an execution trace of the $n$-body simulation application, executing 16 force calculation and particle movement steps. Over $95 \%$ of the time is spent on force calculation tasks that without renaming would be limited to at most 16 concurrent tasks. Thanks to renaming, we can see that all 64 threads are executing tasks during the whole execution. There is an exception for thread 1, which executes no tasks in the beginning of the execution since it is busy creating and submitting the tasks. During this period, it can be seen (but only just barely) that thread 5 executes its tasks faster since that thread shares the floating point unit with thread 1. The dependencies between the time steps can be seen by small gaps in the schedule between most time steps. Running on 64 threads on the Bulldozer system was about 35 times faster than our best serial implementation. Since the system has only 32 floating point units, and the application is dominated by floating point calculations, a speedup much higher than 32 cannot be expected. 


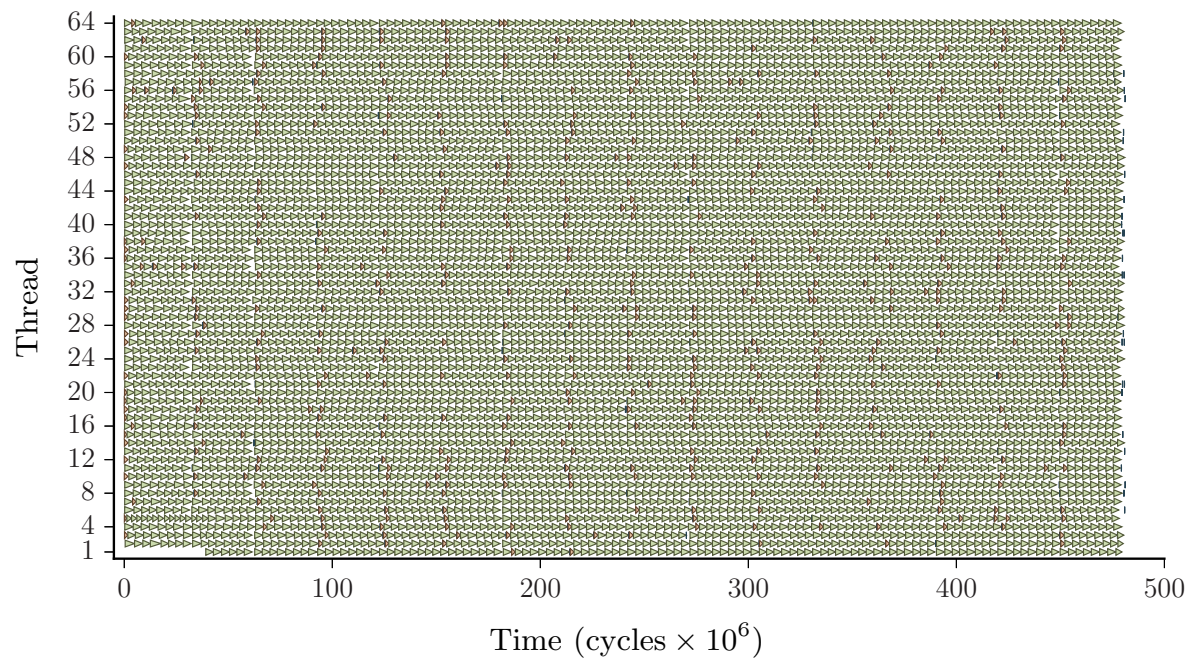

FIG. 9. Execution trace of an n-body simulation of 8192 particles blocked into blocks of 256 particles, stepped for 16 time steps.

6.2. Shallow water simulation. We have previously used SuperGlue to implement a shallow water simulation code [26]. Just like the $n$-body simulation, this is a time dependent problem, where each time step depends on the previous one. It uses the fourth order Runge-Kutta method for time stepping, where each function evaluation includes two sparse matrix-vector multiplications, which is where over $90 \%$ of the execution time is spent.

The problem size is 655,362 unknowns, and the matrices have 20,316,222 nonzeros each. For parallelization, the solution vector is sliced into 131 blocks of 5,000 elements each, except for the last one, which is slightly larger. The matrices are blocked in tiles to match the vector blocks. The nonzeros are centered along the diagonal, so many off-diagonal tiles are empty and discarded, leaving only 391 nonempty tiles. The number of nonzeros varies significantly between different tiles.

In order to handle large numbers of time steps, it is not feasible to submit all the tasks at once. Instead, we introduce a GenerateTask task that submits all tasks needed to take one time step. At the end of each GenerateTask task, it submits another copy of itself which depends on one of the tasks from the time step. Initially, five of these GenerateTask tasks are submitted, in order to generate tasks to take the first five time steps. When each time step is finished, a new GenerateTask task will have been submitted. This way, tasks will be submitted continuously. This continues until the end time of the simulation is reached, after which the GenerateTask tasks will do nothing.

Figure 10 shows the start of an execution trace of the shallow water simulation. The GenerateTask tasks are seen as longer than the others, and the first five are executed by thread 9. More GenerateTask tasks are seen throughout the trace on other threads. Tasks working on different time steps have different colors, and the trace shows that tasks from different time steps are mixed freely. Since SuperGlue is driven by locality, it prefers taking the next time step as soon as possible rather than finishing all tasks from a time step first. When the last task from the first time step is done in this trace, the first tasks from the 11th time step have already been executed. 


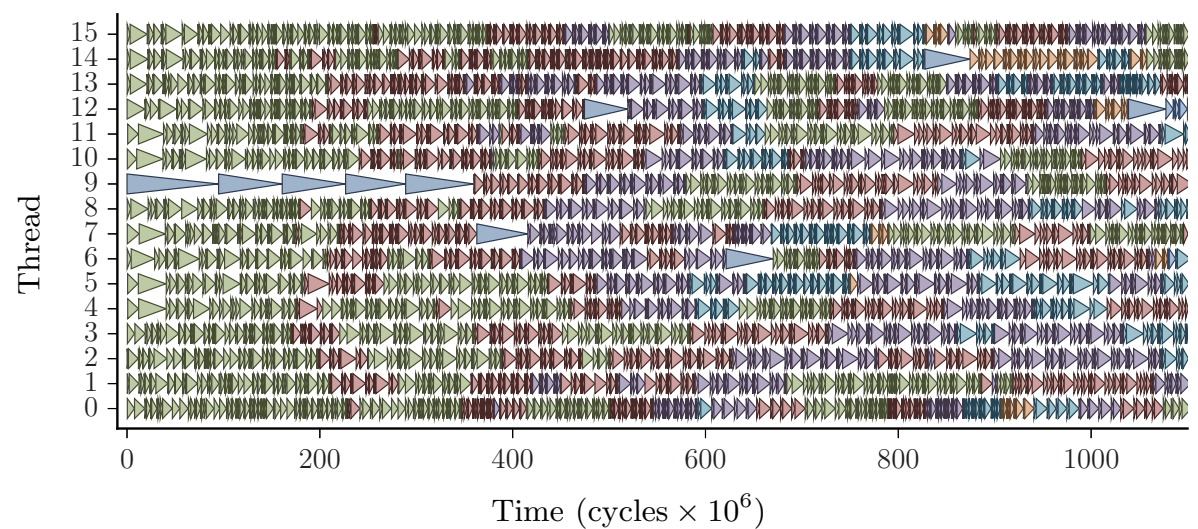

FIG. 10. Part of an execution trace of a shallow water equation solver. Different colors (seen online only) represent tasks working on different time steps.

TABLE 1

Compared frameworks.

\begin{tabular}{ll}
\hline Cilk Plus $^{3}$ & SMPSs 2.4 \\
Intel TBB 4.2 & StarPU 1.1.0 \\
OmpSs $^{4}$ & SuperGlue \\
OpenMP $^{5}$ & Swan \\
QUARK 0.9.0 & XKaapi 2.1 \\
\hline
\end{tabular}

Sparse matrix-vector multiplication is a memory bound operation and is difficult to parallelize efficiently on multicore systems. The parallel version was 5 times faster than the best serial when run on a server with two 8-core AMD Opteron 6220 processors. On this architecture, each pair of cores shares a single floating point unit, and the ideal speedup is therefore 8 . In this run, less than $2 \%$ of the execution time was spent on task management (including executing the task generation tasks) or on threads being idle. The reason the speedup is not closer to 8 is due to contention when all threads compete for the memory bandwidth.

7. Comparison with other efforts. In order to verify that our programming model is competitive, we perform a number of performance experiments comparing our implementation against other efforts. For this, we use microbenchmarks designed to stress the runtime systems and to produce results that are as generalizable as possible. These benchmarks are available at GitHub. ${ }^{7}$

All the experiments measure the time from when the first tasks start to when the last task finishes. This includes both the overhead induced by task management and the quality of the scheduling, but it excludes the time to start up and shut down the runtime system. As in the experiments in section 5, we let each task wait for a number of cycles instead of performing actual computations, in order to isolate the task management from application-specific behavior. The frameworks we have compared are listed in Table 1 . They were all compiled with the -O3 optimization

\footnotetext{
${ }^{3}$ Using the Cilk Plus branch from GCC 4.8

${ }^{4}$ Built using Nanos++ 0.7a (2013-09-05) and Mercurium 1.99.0 (2013-09-10)

${ }^{5}$ Compiled with GCC 4.7.2 on Bulldozer and GCC 4.8.1 on Desktop

${ }^{6}$ Built from latest version on GitHub, last commit 2013-08-10

${ }^{7}$ https://github.com/tillenius/tasklib-comparison
} 


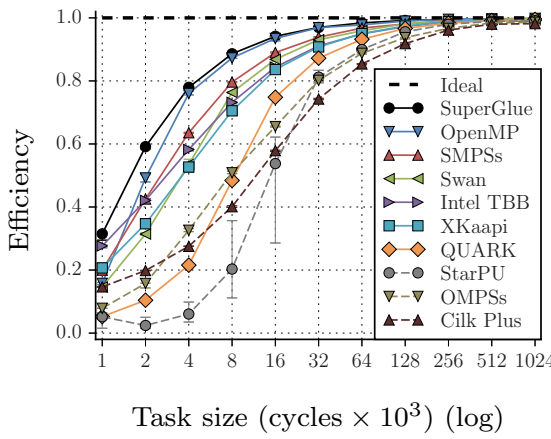

(a) 4 cores

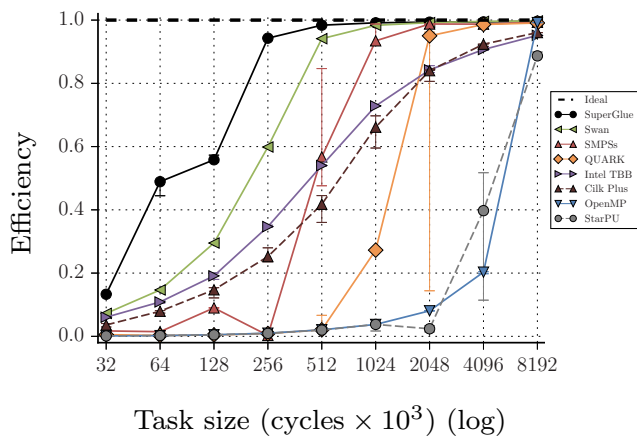

(b) 64 cores

FIG. 11. Independent tasks: comparison of efficiency between different task frameworks. Error bars show the upper and lower quartiles.

flag. The $-\mathrm{O} 2$ level was evaluated too, but there was no qualitative difference, and $-\mathrm{O} 3$ was selected.

The default settings were used for all frameworks, except for StarPU, which issues a warning that the user should select a scheduling strategy. All experiments with StarPU use the work stealing scheduler, which was found out to give the best results in an initial experiment.

7.1. Independent tasks. This benchmark investigates the behavior when there are no dependencies between the tasks. The experiment is basically the same as in section 5.2, except that task allocation is now included for SuperGlue and that we always run on all available CPUs. Each task only waits for a predefined number of cycles, and the time to submit and execute all tasks is measured. The test was executed on two different machines, the 64-core Bulldozer server (described in section 5.2) and a 4-core desktop machine called Desktop, which is equipped with an Intel Core i7 $2600 \mathrm{~K}$ processor running at $3.4 \mathrm{GHz}$.

In this benchmark, we create 12,800 tasks when running on 64 threads, and 2400 tasks when running on 4 threads, which turned out to be enough work for all threads and avoids anomalies at startup and shutdown. We repeat the experiment for different number of cycles in each task wait and plot the efficiency, defined to be the ratio of the ideal execution time (number of tasks $\times$ cycles each task waits / number of CPUs) to the measured execution time. This gives a measure of how large tasks must to be in order to make task management negligible and the execution efficient. For each wait time, the experiment was repeated 50 times on Desktop and 5 times on Bulldozer in order to capture dispersion in execution time.

Figure 11 shows the outcome. SuperGlue is the most efficient framework on both 4 and 64 cores. OpenMP is about as efficient as SuperGlue on 4 cores but ended up among the least efficient on 64 cores. We see here that StarPU is among the frameworks which require the largest task sizes in order to reach high efficiency. An explanation is that StarPU targets heterogeneous platforms, and its main strength is deciding whether tasks should run on the GPU or CPU. Since we only consider homogeneous CPUs here, spending extra time on scheduling is not beneficial.

7.2. Tasks with dependencies. In order to compare the performance of the dependency management and scheduling, we use the dependencies from a block Cholesky 


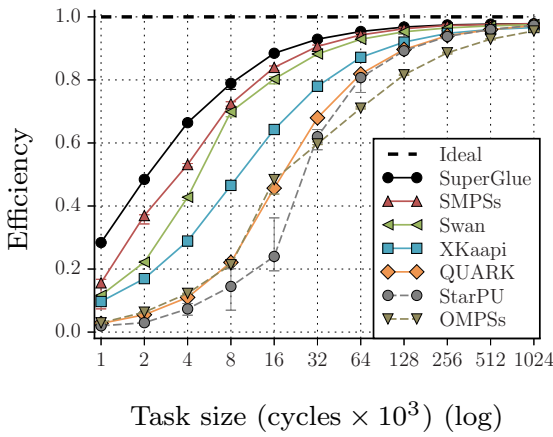

(a) 4 cores

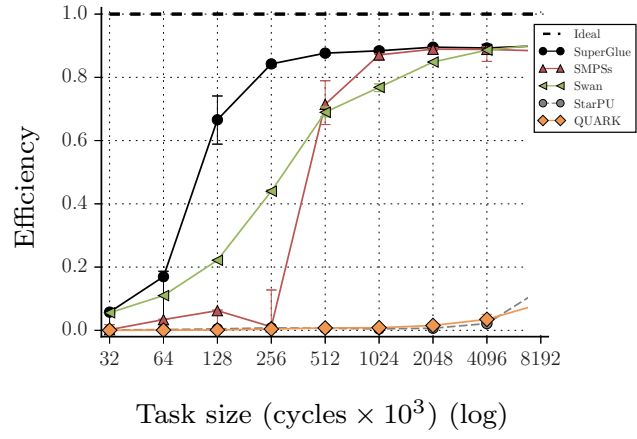

(b) 64 cores

FIG. 12. Tasks with dependencies: comparison of efficiency between different task frameworks. Error bars show the upper and lower quartiles.

factorization, which is selected because it is a standard benchmark to represent realistic dependencies. In this experiment, we make use of data-driven dependencies and frameworks that do not provide this; i.e., OpenMP, Intel TBB, and Cilk Plus are not included. Again, the tasks perform no actual work but only wait for a determined number of cycles.

The experiments are run both on the 4-core Desktop machine, where the matrix is blocked into $20 \times 20$ tiles, and on the 64-core Bulldozer server, where it's blocked into $50 \times 50$ tiles. This corresponds to 1,540 tasks on 4 cores and 22,100 on 64 cores. The test was repeated 20 times on Desktop and 10 times on Bulldozer in order to capture dispersion in execution time.

Figure 12 shows the results. SuperGlue reaches high efficiency for smaller task sizes than other frameworks. On 4 cores, the results are similar to the results from the comparison with independent tasks, but all frameworks have slightly lower efficiency. This is both because of dependency management costs and because the dependencies limit the parallelism. The ideal efficiency does not take task dependencies into account, so the best possible efficiency is actually slightly lower. On 64 cores, results are also similar, except for QUARK and StarPU, which now require much larger tasks to reach good efficiency. When moving from 8,000 tasks to 16,000 tasks, there is a jump in the figure for StarPU, and the overhead doubles. In the experiments on the 64-core Bulldozer server in sections 7.1 and 7.2, 12,800 (respectively, 22,100) tasks were submitted, which is just around this jump, and this is part of the explanation of why StarPU did not reach higher efficiency in the previous tests.

7.3. Scaling with number of tasks. To verify that our solution scales with the number of tasks, this benchmark submits different numbers of tasks, each waiting for 100,000 cycles, and measures how much time is spent on overhead. The overhead is the extra time it takes to submit and execute all tasks over the ideal time $(100,000$ cycles per task $\times$ number of tasks / 4 cores).

The results are shown in Figure 13. Most frameworks are not affected by the number of tasks. The runtime for OmpSs varies for low numbers of tasks but reaches a steady level for larger numbers of tasks. StarPU is the only framework that is sensitive to large numbers of tasks. When moving from 8,000 tasks to 16,000 tasks, the overhead is doubled. SuperGlue scales well with the number of tasks and has the lowest overhead among all compared frameworks. 


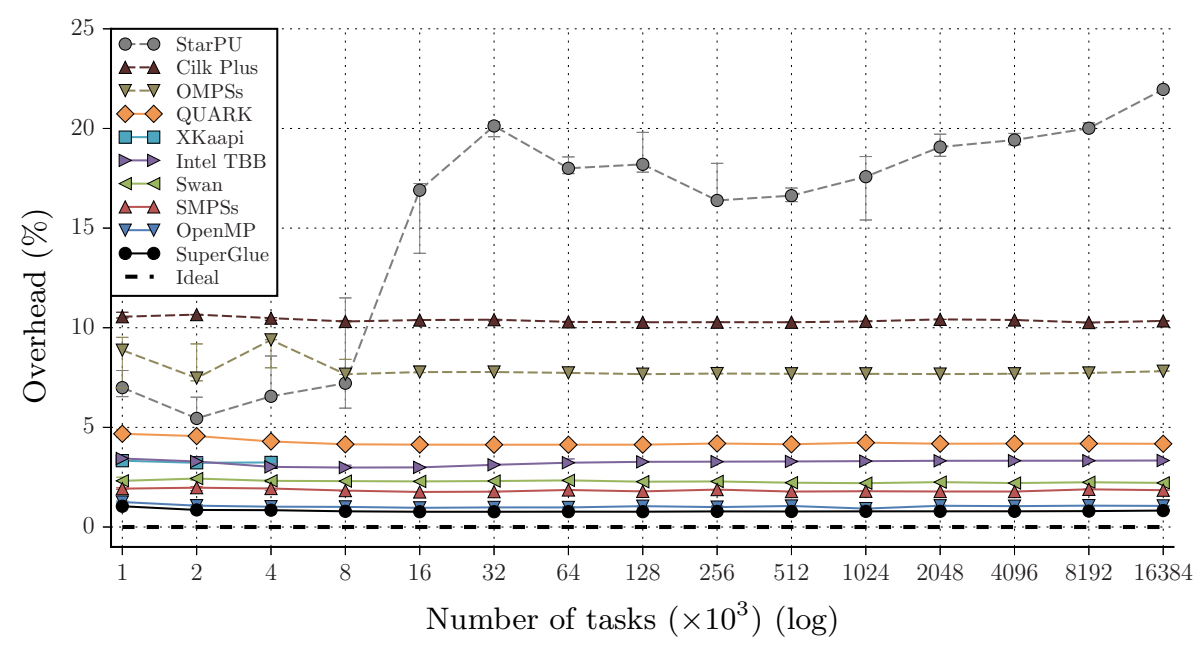

FIG. 13. Scaling with respect to the number of tasks when running on 4 cores. Error bars show the first and third quartiles of five executions.

8. Comparison with ACML. To verify that the task-based approach is competitive against other parallelization strategies, we return to the Cholesky benchmark. In this section we present a comparison of the implementation outlined in Listing 1 against the multithreaded Cholesky factorization available in AMD Core Math Library (ACML) version 6.1.0.31. ACML was selected since the comparison is performed on a server with two 8-core AMD Bulldozer processors (Opteron 6220) running at $3 \mathrm{GHz}$, for which ACML is highly optimized. It represents a highly regarded third-party product that is optimized for performance and free to use any suitable method for parallelization.

The Cholesky factorization of a matrix of size $8,000 \times 8,000$ is computed. In the multithreaded ACML version, this is a single library function call. The SuperGlue implementation divides the matrix into $25 \times 25$ tiles of $320 \times 320$ elements each and uses computational kernels from the nonthreaded version of ACML. How the tile sizes are decided and how this affects performance are not in the scope of this experiment, but the rule of thumb is that having larger tiles means better performance in the kernels, while having more tiles allows more parallelism. When the tiles are large enough and there is enough parallelism, the performance is not sensitive to the choice of tile sizes.

To make the comparison fair, the timing includes allocating memory for the tiles, dividing the input matrix into smaller tiles, and starting the SuperGlue runtime system, as well as combining the results back together to overwrite the input matrix, shutting down the runtime system, and releasing all allocated memory. The source code for this benchmark is available from GitHub. ${ }^{8}$

The experiment was run 20 times, and the median and interquartile range is presented in Table 2. The version parallelized with SuperGlue is found to be $50 \%$ more efficient than the multithreaded version provided by ACML. This shows the strength of the dependency-driven task-based approach for this problem.

\footnotetext{
${ }^{8}$ https://github.com/tillenius/sg-blas-comparison
} 
TABLE 2

Comparison between SuperGlue and threaded ACML for multithreaded Cholesky factorization.

\begin{tabular}{ll}
\hline Implementation & Performance (interquartile range) \\
\hline ACML (nonthreaded) & 13.8 GFlops (13.8-14.0) \\
ACML (threaded) & 80.1 GFlops (78.5-80.3) \\
SuperGlue+ACML & 120.6 GFlops (119.2-124.6) \\
\hline
\end{tabular}

9. Discussion and future work. The most promising approach to programming for multicore processors is to divide the software into tasks and let a runtime system schedule these tasks for execution in parallel. In real applications, the tasks have dependencies, and these dependencies must be handled correctly and efficiently. Introducing artificial dependencies or unnecessary synchronization can be devastating for performance. Instead, fine-grained synchronization is needed. When the granularity is small, task scheduling must be fast, or the scheduling overhead will dominate the execution time and prevent the software from scaling at all.

In this paper we have presented a task-based programming model where the user specifies dependencies between tasks by creating handles that represent shared resources and declaring which handles each task accesses. Handles have version numbers, and tasks are ready to execute when certain versions of these handles are available. This model is convenient and flexible for the user and introduces no artificial synchronizations. We have also presented an implementation of this system called SuperGlue. In this implementation, task scheduling is distributed, it requires no central information, and it is driven by locality. Our implementation is shown to be efficient, to scale well, and to be practically applicable. We compare our implementation against other task-based systems, as well as against highly optimized third-party code, and show that it performs equally well or better.

We are currently working on extending the SuperGlue model to support distributed memory parallelism via MPI. A working prototype, implemented as a layer on top of SuperGlue, has already been developed and used to build a distributed memory version of the shallow water code presented in section 6.2 , with promising results $[26]$.

\section{REFERENCES}

[1] C. Augonnet, S. Thibault, R. Namyst, and P.-A. Wacrenier, StarPU: A unified platform for task scheduling on heterogeneous multicore architectures, Concurrency Computat. Pract. Exper., Euro-Par 2009, 23 (2011), pp. 187-198.

[2] E. Ayguadé, N. Copty, A. Duran, J. Hoeflinger, Y. Lin, F. Massaioli, X. Teruel, P. Unnikrishnan, And G. Zhang, The design of OpenMP tasks, IEEE Trans. Parallel Distrib. Syst., 20 (2009), pp. 404-418.

[3] P. Bauer, S. Engblom, and S. Widgren, Fast Event-Based Epidemiological Simulations on National Scales, preprint, arXiv:1502.02908 [q-bio.PE], 2014.

[4] P. Bellens, J. M. Pérez, R. M. Badia, and J. Labarta, CellSs: A programming model for the Cell BE architecture, in Proceedings of the 2006 ACM/IEEE Conference on Supercomputing (SC '06), ACM, New York, 2006.

[5] R. D. Blumofe, C. F. Joerg, B. C. Kuszmaul, C. E. Leiserson, K. H. Randall, and Y. ZHOU, Cilk: An efficient multithreaded runtime system, SIGPLAN Not., 30 (1995), pp. 207-216.

[6] A. Buttari, J. Langou, J. Kurzak, and J. Dongarra, A class of parallel tiled linear algebra algorithms for multicore architectures, Parallel Comput., 35 (2009), pp. 38-53.

[7] L. Dagum and R. Menon, OpenMP: An industry standard API for shared-memory programming, IEEE Comput. Sci. Eng., 5 (1998), pp. 46-55.

[8] J, Dongarra, J. Kurzak, J. Langou, J. Langou, H. Ltaief, P. Luszczek, A. YarKhan, W. Alvaro, M. Faverge, A. Haidar, J. Hoffman, E. Agullo, A. Buttari, and 
B. HADRI, PLASMA Users' Guide, http://icl.cs.utk.edu/plasma/.

[9] A. Duran, E. Ayguadé, R. M. Badia, J. Labarta, L. Martinell, X. Martorell, and J. Planas, OmpSs: A proposal for programming heterogeneous multi-core architectures, Parallel Process. Lett., 21 (2011), pp. 173-193.

[10] F. Galilée, J.-L. Roch, G. G. H. Cavalheiro, and M. Doreille, Athapascan-1 : On-line building data flow graph in a parallel language, in Proceedings of the 1998 International Conference on Parallel Architectures and Compilation Techniques (PACT '98), Washington, D.C., 1998, IEEE Computer Society, Los Alamitos, CA, pp. 88-95.

[11] T. Gautier, J. V. F. Lima, N. Maillard, and B. Raffin, XKaapi: A runtime system for data-flow task programming on heterogeneous architectures, in Proceedings of the 27th International IEEE Symposium on Parallel and Distributed Processing (IPDPS '13), 2013, pp. $1299-1308$.

[12] M. Holm, S. Engblom, A. Goude, And S. Holmgren, Dynamic autotuning of adaptive fast multipole methods on hybrid multicore CPU and GPU systems, SIAM J. Sci. Comput., 36 (2014), pp. C376-C399.

[13] Intel Cilk Plus, http://www.cilkplus.org/.

[14] Intel Threading Building Blocks, http://threadingbuildingblocks.org/.

[15] J. KuRzaK AND J. DongarRa, Implementing linear algebra routines on multi-core processors with pipelining and a look ahead, in Applied Parallel Computing: State of the Art in Scientific Computing, Lecture Notes in Comput. Sci. 4699, B. Kågström, E. Elmroth, J. Dongarra, and J. Waśniewski, eds., Springer, Berlin, Heidelberg, 2007, pp. 147-156.

[16] J. Kurzak, H. Ltaief, J. Dongarra, and R. Badia, Scheduling dense linear algebra operations on multicore processors, Concurr. Comput., 22 (2010), pp. 15-44.

[17] M. S. Lam And M. C. Rinard, Coarse-grain parallel programming in Jade, SIGPLAN Not., 26 (1991), pp. 94-105.

[18] C. E. Leiserson, The Cilk++ concurrency platform, J. Supercomput., 51 (2010), pp. 244-257.

[19] C. Niethammer, C. W. Glass, and J. Gracia, Avoiding serialization effects in data / dependency aware task parallel algorithms for spatial decomposition, in Proceedings of the 10th IEEE International Symposium on Parallel and Distributed Processing with Applications (ISPA '12), Washington, D.C., 2012, IEEE Computer Society, Los Alamitos, CA, pp. $743-748$.

[20] OpenMP Architecture Review Board, OpenMP Application Program Interface Version 4.0, 2013.

[21] J. M. PÉRez, R. M. BAdia, and J. LABARta, A dependency-aware task-based programming environment for multi-core architectures, in Proceedings of the 2008 IEEE International Conference on Cluster Computing, 2008, pp. 142-151.

[22] L. Sunde, Parallelizing a Software Framework for Radial Basis Function Methods, manuscript, 2011.

[23] M. Tillenius, Leveraging Multicore Processors for Scientific Computing, licentiate thesis, Department of Information Technology, Uppsala University, Uppsala, Sweden, 2012.

[24] M. Tillenius And E. Larsson, An efficient task-based approach for solving the $n$-body problem on multicore architectures, in PARA 2010: State of the Art in Scientific and Parallel Computing, University of Iceland, Reykjavík, Iceland, 2010.

[25] M. Tillenius, E. Larsson, R. M. Badia, and X. Martorell, Resource-aware task scheduling, ACM Trans. Embed. Comput. Syst., 14 (2015), pp. 5:1-5:25.

[26] M. Tillenius, E. Larsson, E. Lehto, And N. Flyer, A scalable RBF-FD method for atmospheric flow, J. Comput. Phys., 298 (2015), pp. 406-422.

[27] H. Vandierendonck, G. Tzenakis, and D. S. Nikolopoulos, A unified scheduler for recursive and task dataflow parallelism, in Proceedings of the 2011 International Conference on Parallel Architectures and Compilation Techniques (PACT '11), 2011, pp. 1-11.

[28] A. YarKhan, J. Kurzak, and J. Dongarra, QUARK Users' Guide: Queueing and Runtime for Kernels, Tech. Report ICL-UT-11-02, Innovative Computing Laboratory, University of Tennessee, Knoxville, TN, 2011.

Copyright $@$ by SIAM. Unauthorized reproduction of this article is prohibited. 